\title{
Comparison of outcome of closed reduction and percutaneous pinning and, open reduction and internal fixation with k-wire in Gartland extension type III supracondylar fracture of distal humerus in pediatric population
}

\author{
KC Bhogendra Bahadur ${ }^{1}$, Lamichhane Norman ${ }^{1}$, Mishra Chandra Bahadur ${ }^{1}$, Khatri Bharat Bahadur ${ }^{1}$, \\ Dhakal Sabita ${ }^{2}$ \\ ${ }^{1}$ Department of Orthopedics, Pokhara Academy of Health Sciences, Pokhara, Western Regional Hospital, \\ ${ }^{2}$ Nutrition and SBCC Officer Suaahara
}

\section{*Corresponding author}

Dr. Bhogendra Bahadur KC, Senior Consultant,

Department of Orthopedics,

Pokhara Academy of Health

Sciences,

Western Regional Hospital, Pokhara

Email:bhogendrakc@gmail.com

Article received : February 4, 2018

Article accepted : March 7, 2018

\begin{abstract}
Background : Supracondylar fracture of the distal humerus is one of the commonest fracture in pediatric age group. Though there is consensus of treating type III fracture operatively, no study has compared the outcome between Closed Reduction and Percutaneous Pinning (CRPP) and Open Reduction and Internal Fixation (ORIF) with k-wire in our setup. Materials and Methods: Retrospective comparison study was done on eighty seven cases of Type III supracondylar fracture of distal humerus underwent operative procedure. Fifty four (54) cases underwent CRPP and 33 cases were managed with ORIF with k-wire, and they were followed up till 6 months post-operatively. Results : The mean time for radiological union in patient who underwent CRPP was $4.37 \pm 0.94$ weeks and that for the patient who underwent ORIF was $4.45 \pm 0.13$ weeks, the difference of which was statistically insignificant (p-value $>0.05$ ). $83.3 \%$ of CRPP group and $78.8 \%$ in ORIF group had excellent functional outcome and only $3 \%$ in ORIF group had poor functional outcome.Conclusion : Though both the group don't have significant advantage of functional outcome among each other CRPP with limited attempt should be preferred to ORIF with k-wire for the advantage of avoiding surgical scar and reducing surgery time and exposure to anaesthetic agents.
\end{abstract}

Key Words: Fracture, closed reduction, percutaneous fixation

\section{INTRODUCTION}

Supracondylar fracture of distal humerus is the most common pediatric fracture around elbow visiting emergency room. ${ }^{1,2}$ The Gartland classification system is the most commonly used classification to describe extension-type supracondylar fractures in which Type I fractures are nondisplaced, Type II fractures have an intact posterior hinge, and Type III fractures involve complete displacement. ${ }^{3}$ There has been trend towards operative treatment now a days in Type II and Type III fractures. ${ }^{4,5}$ Most of the literature support closed reduction and percutaneous pinning as the treatment of choice for Type III fractures with ORIF reserved for irreducible fracture. ${ }^{1,6}$ Complications like perioperative nerve palsy, hardware complications etc, are higher with Type III fractures . ${ }^{7}$ Functional outcome is poor with posterior approach as compared to medial, lateral or anterior approach but has fair cosmetic outcome. ${ }^{8}$

\section{MATERIALS AND METHODS}

Eighty seven cases of Gartland extension Type III supracondylar fractures of distal humerus in the age group of 4 to 14 years treated at Kaski Model Hospital from year 2011 March to 2016 August were included in the study. The cases with previous history of humerus fracture and open fractures were excluded from the study. Fifty four cases underwent closed reduction and percutaneous pinning (CRPP), and thirty three cases with failed closed reduction underwent open reduction and internal fixation (ORIF). All the cases were taken up for the procedure after obtaining written consent for intravenous or general anaesthesia. Both the CRPP and ORIF cases were fixed with 2 cross k-wires. CRPP was done under the guidance of image intensifier. The approach for all ORIF cases was posterior triceps splitting approach after isolating and securing the ulnar nerve 
in a lateral decubitus position with forearm hanging and the arm resting on an arm rest. Both group of cases were supported with long arm posterior slab till the radiological signs of union was noted and followed up at 2 weeks, 4 weeks, 6 weeks, 3 months and 6 months. The pins were removed after radiological signs of union and active physiotherapy was started. Function was assessed using Flynn's criteria at 6 months of follow up.

\section{RESULTS}

Eighty seven cases of Gartland extension Type III supracondylar fracture of distal humerus were included in our study in which 54 cases underwent for CRPP and 33 cases underwent ORIF with k-wire. 59 cases (68\%) were male patient and 28 cases(32\%) were female patient. The mean age of patient in CRPP group was 7.8 years and in ORIF group was 8 years ( 7.9 years overall) which was statistically not significant in difference of age.

The mean time for radiological union in patient who underwent CRPP was $4.37 \pm 0.94$ weeks and that for the patient who underwent ORIF was $4.45 \pm 0.13$ weeks.

Figure 1: Preoperative and post-union images of CRPP group
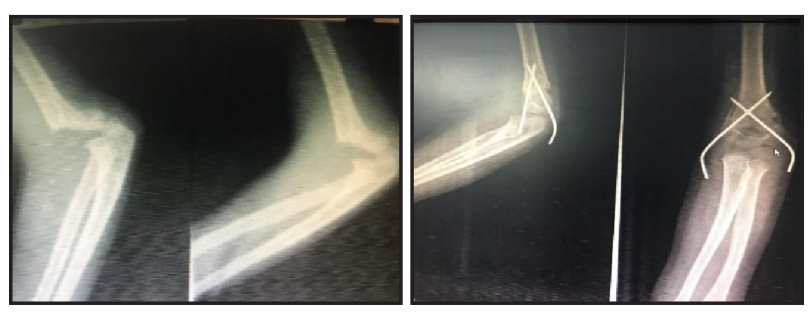

Figure 2: Preoperative, post-operative, and post-union images of ORIF group
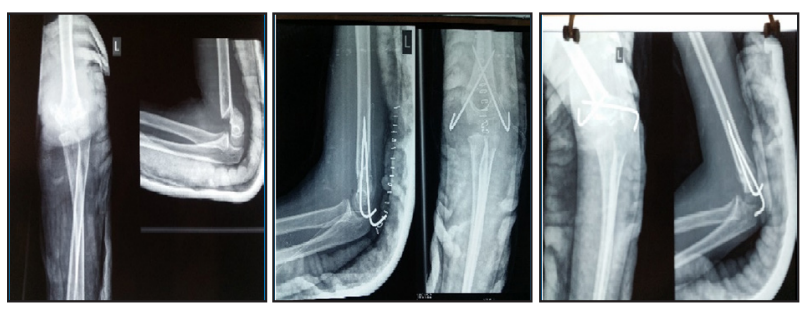

Most of the patient in both group achieved normal range of motion [45 (83.3\%) in CRPP group and 26 $(78.8 \%)]$ and the remaining had extensor lag of less than 15 degree except 1 patient $(3 \%)$ in ORIF group who had extensor lag of 20 degree (table 1). Corrective osteotomy was performed in this patient after 6 months of treatment. Loss of carrying angle was less than 5 degree in all the cases.

Table 1: Functional outcome according to Flynn criteria

\begin{tabular}{lll}
\hline Outcome & CRPP & ORIF \\
\hline Excellent & $83.3 \%$ & $78.8 \%$ \\
Good & $11.1 \%$ & $12.1 \%$ \\
Fair & $5.6 \%$ & $6.1 \%$ \\
Poor & $0 \%$ & $3 \%$ \\
\hline
\end{tabular}

We had 6 cases of median nerve injury (all in CRPP group) and 4 cases ( 2 in CRPP and 2 in ORIF) of radial nerve injury. All cases recovered completely at 6 weeks. 2 cases in ORIF group underwent extended posterolateral approach to confirm the intactness of radial nerve. Three $(3.4 \%)$ of total case had pulseless hand in whom pulse returned after treatment (CRPP was possible in all cases). Six patients (11\%) in CRPP group and $3(9 \%)$ in ORIF group had pin tract infection as complication which got better with dressing and oral antibiotic in all cases. Three patients (5.6\%) in CRPP group had ulnar nerve injury which recovered spontaneously at 6 weeks in 2 cases and at weeks in 1 case with the removal of medial pin immediately after the injury was confirmed.

\section{DISCUSSION}

No single treatment protocol is suitable for all the types of supracondylar fracture. The widely accepted treatment protocol for Gartland extension Type III supracondylar fracture is closed reduction and percutaneous pinning, with open reduction reserved for failed closed reduction. There is more advantage of closed reduction over open reduction, but the comparison of these two different treatment protocols has been done in very few studies. Thirty seven percentage (33 out of 87) of our cases required ORIF as compared to $45 \%$ (14 out of 31 ) in study done by O'Hara et al. ${ }^{9}$ ORIF has been reported in the range of $1.3 \%$ to $46 \%$ in different studies. ${ }^{10-13} \mathrm{CRPP}$ is preferred when closed reduction is successful at first at- 
tempt. ${ }^{14}$ Repeated attempt of manipulation is associated with increased number of complications and increased surgical time as most of the fractures are irreducible due to the interposition of brachialis muscle, median nerve, or brachial artery. ${ }^{15}$ Time from injury to surgery has been an issue of controversy regarding its effect on complication as well as open reduction rates. ${ }^{8}$

The radiological union in most of the studies ${ }^{8}$ took 4 to 5 weeks similar to our study in both groups $(4.37 \pm 0.94$ weeks in CRPP group and $4.45 \pm 0.13$ in ORIF group). The difference of union time in two groups is statistically insignificant in our study meaning that the method of treatment doesn't have influence on the union time of the fracture.

There is no clear evidence in the literature regarding which of the surgical approaches could bring about the best functional and cosmetic outcomes, as well as less complications. ${ }^{8}$ Anterior approach is preferred for the cases with neurovascular injury due advantage of better exposure of such structures. The functional outcome shows a high frequency of excellent results with the lateral and medial approaches and a high frequency of good results with the anterior approach though the time to union is not affected by the type of surgical approach used. ${ }^{8}$ However, our study shows that excellent functional result can be achieved with posterior approach. Some authors have demonstrated no correlation between stiffness and the type of surgical approach used, especially regarding the posterior approach. ${ }^{16}$ No surgical approach showed a higher incidence of complications such as compartment syndrome, nonunion, or nerve/vascular injury.

Iatrogenic nerve injury was seen in 3 patients $(5.6 \%)$ in CRPP group in our study similar to other literature ${ }^{17}$ reporting of $4 \%$. With regard to vascular injury, 10-20\% of patients with a Type III supracondylar fracture present with an absent pulse. ${ }^{18,19}$ But in our case we had only $3(3.4 \%)$ such cases but pulse returned with closed reduction in all 3 cases.

Limitation of our study is being a retrospective study. A randomized control trial could have more firm established conclusion. Ours study group didn't have uniform distribution of preoperative complications. Long term follow up would be valuable to establish the result regarding function.

\section{CONCLUSION}

Type III supracondylar fracture should be treated with k-wire fixation in pediatric population. Closed or open reduction doesn't have significant advantage on function and union rate among one another. However CRPP with limited attempt should be preferred to ORIF with $\mathrm{k}$-wire for the advantage of avoiding surgical scar and reducing surgery time and exposure to anaesthetic agents. Medial pinning should be done carefully during CRPP to reduce the iatrogenic injury to ulnar nerve. ORIF with posterior approach is an easier approach without any difference in functional outcome.

\section{REFERENCES}

1. Otsuka NY, Kasser JR. Supracondylar fractures of the humerus in children. Journal of the American Academy of Orthopaedic Surgeons. 1997; 5(1):19-26

2. Cheng JC, Shen W. Limb fracture pattern in different pediatric age groups: a study of 3,350 children. Journal of orthopaedic trauma. 1993; 7(1):15-22

3. Gartland JJ. Management of supracondylar fractures of the humerus in children. Surg Gynecol Obstet. 1959

4. Bashyal RK, Chu JY, Schoenecker PL, Dobbs MB, Luhmann SJ, Gordon JE. Complications after pinning of supracondylar distal humerus fractures. Journal of Pediatric Orthopaedics. 2009; 29(7):7048

5. Camus T, MacLellan B, Cook PC, Leahey JL, Hyndman JC, El-Hawary R. Extension type II pediatric supracondylar humerus fractures: a radiographic outcomes study of closed reduction and cast immobilization. Journal of Pediatric Orthopaedics. 2011; 31(4):366-71

6. Kasser J. Percutaneous pinning of supracondylar fractures of the humerus. Instructional course lectures. 1992; 41:385-90

7. Oetgen ME, Mirick GE, Atwater L, Lovejoy JF. Complications and predictors of need for return to the operating room in the treatment of supracondylar humerus fractures in children. The open orthopaedics journal. 2015; 9:139

8. Pretell Mazzini J, Rodriguez Martin J, Andres Esteban EM. Surgical approaches for open reduction and pinning in severely displaced supracondylar humerus fractures in children: a systematic review. Journal of children's orthopaedics. 2010; 4(2):143-52

9. O'hara L, Barlow J, Clarke NM. Displaced supracondylar fractures of the humerus in children. Bone \& Joint Journal. 2000; 82(2):204-10 10. Gupta N, Kay RM, Leitch K, Femino JD, Tolo VT, Skaggs DL. Effect of surgical delay on perioperative complications and need for open reduction in supracondylar humerus fractures in children. Journal of Pediatric Orthopaedics. 2004;24(3):245-8

11. Damelsson L, Petterssox H. Open reduction and pin fixation of severely displaced supracondylar fractures of the humerus in children. Acta Orthopaedica Scandinavica. 1980; 51(1-6):249-55 